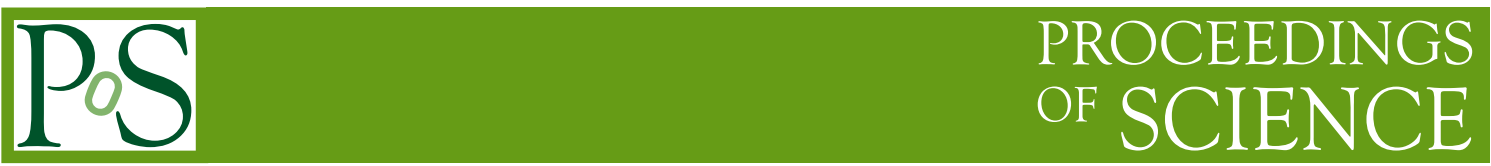

\title{
The CMS RPC performance and simulation
}

\section{Roumyana Hadjiiska* On behalf of the CMS Collaboration}

University of Sofia,

Faculty of Physics, 5 James Bourchier Blvd., 1164 Sofia, Bulgaria

E-mail: roumyana.mileva.hadjiiska@cern.ch

The performance of the Resistive Plate Chamber (RPC) subsystem of CMS (Compact Muon Solenoid) experiment has been studied using cosmic-ray data and proton-proton collision data at $\sqrt{s}=7 \mathrm{TeV}$. The experimental data are used for realistic RPC simulation parameters estimation. An algorithm for simulation of the RPC response is implemented in the CMSSW (CMS software framework) and a dedicated validation procedure is developed. A good agreement between the simulated and experimental data is achieved.

XI workshop on Resistive Plate Chambers and Related Detectors - RPC2012,

February 5-10, 2012

INFN Laboratori Nazionali di Frascati Italy

\footnotetext{
* Speaker.
} 


\section{The CMS Muon system}

The main subsystems of the CMS (Compact Muon Solenoid) detector include the tracker system, the calorimetric system, which are located inside the $3.8 \mathrm{~T}$ superconducting solenoid and the muon system, which is situated in the return yoke of the solenoidal magnetic field of $1.8 \mathrm{~T}$ [1].

The CMS muon system is composed of three types of gaseous particle detectors. The central barrel part has a cylindrical shape and it is subdivided in five wheels. There are four muon stations at different radii built in the barrel. They consist of Drift Tubes (DT) which are complemented by six layers of Resistive Plate Chambers (RPC). There are four muon stations in each of the end-caps. They are built of Cathode Strip Chambers (CSC) complemented by the RPC in the inner three endcap stations. The RPC system consists of 480 barrel chambers and 432 end-cap chambers and it covers a geometrical region up to $|\eta|<1.6$, where $\eta$ is the pseudo-rapidity.

CMS uses double-gap RPCs operating in avalanche mode [2]. A double-gap consists of two single gaps which are put one on the top of the other with common read-out copper strips in the middle. A signal induced by two simultaneous avalanches leads to an improved detection efficiency as it is the sum of the two single gap signals. Each chamber consists of two or three adjacent double-gaps held together by aluminum profiles. There are 1020 double-gaps in the barrel and 1296 in the end-caps. Each double-gap has up to 96 read-out strips. There are more than 100 000 read-out channels in total for the whole RPC subsystem. The chambers in the barrel have rectangular geometry and the pitch of the strips varies from $2.3 \mathrm{~cm}$ for the first muon station to 4.1 $\mathrm{cm}$ for the fourth muon station. The chambers in the end-caps have trapezoidal geometry and the pitch of the strips varies from $1.7 \mathrm{~cm}$ at the lowest radii to roughly $3.6 \mathrm{~cm}$ at the highest radii.

\section{The RPC response simulation}

CMSSW is a dedicated software framework for CMS event reconstruction, Monte Carlo (MC) simulation and data analysis. The simulation of the RPC response is a necessary part of the full CMSSW simulation chain which is used for the physical CMS analysis. The simulation chain in CMSSW is organized in the following stages:

- event generation - events are generated by a physics event generators, interfaced by CMSSW.

- event simulation - CMSSW interface to GEANT4[3] is used to simulate the passage of particles through the detector, taking into account the mechanical design, material budget and the magnetic field. In the particular case of RPC simulation, the result from the simulation step is a simulated hit in the active detector volume;

- digitization - in response to each simulated hit one or few consecutive strips are fired with a given probability. Timing information is used to assign the detector response to a given bunch crossing. The intrinsic detector noise is simulated on the same step;

- Level 1(L1) trigger is emulated and High Level Trigger(HLT) algorithms are ran over the simulated events on this step;

- event reconstruction. If necessary, the reconstruction algorithms are ran over the simulated events on this step 
The simulation of the real physical processes inside the RPC is a very complex and computerintensive task. Therefore the RPC digitization is heavily parametrized. In order to tune the MC data to the experimental data it is very important to update regularly the parameters used for the RPC response simulation. The following parameters from a dedicated database are used in the default digitization algorithm:

- efficiency for each readout strip;

- noise rate for each readout strip;

- cluster size distributions;

- timing for each double-gap.

Presently, three sets of parameter values are used:

- the ideal conditions e.g., RPC efficiency 95\%; strip noise $0.05 \mathrm{~Hz} / \mathrm{cm}^{2}$;

- the parameters estimated using cosmic-ray data;

- the parameters estimated from proton-proton collisions data.

Data collected in 2011 after the automatic pressure correction of HV supply has been applied are used for RPC simulation parameters estimation.

A dedicated analysis is used to validate the simulation results and compare them to the data.

\subsection{Efficiency}

The comparison between the efficiency estimated from experimental data and the simulated efficiency is shown on the figure 1.
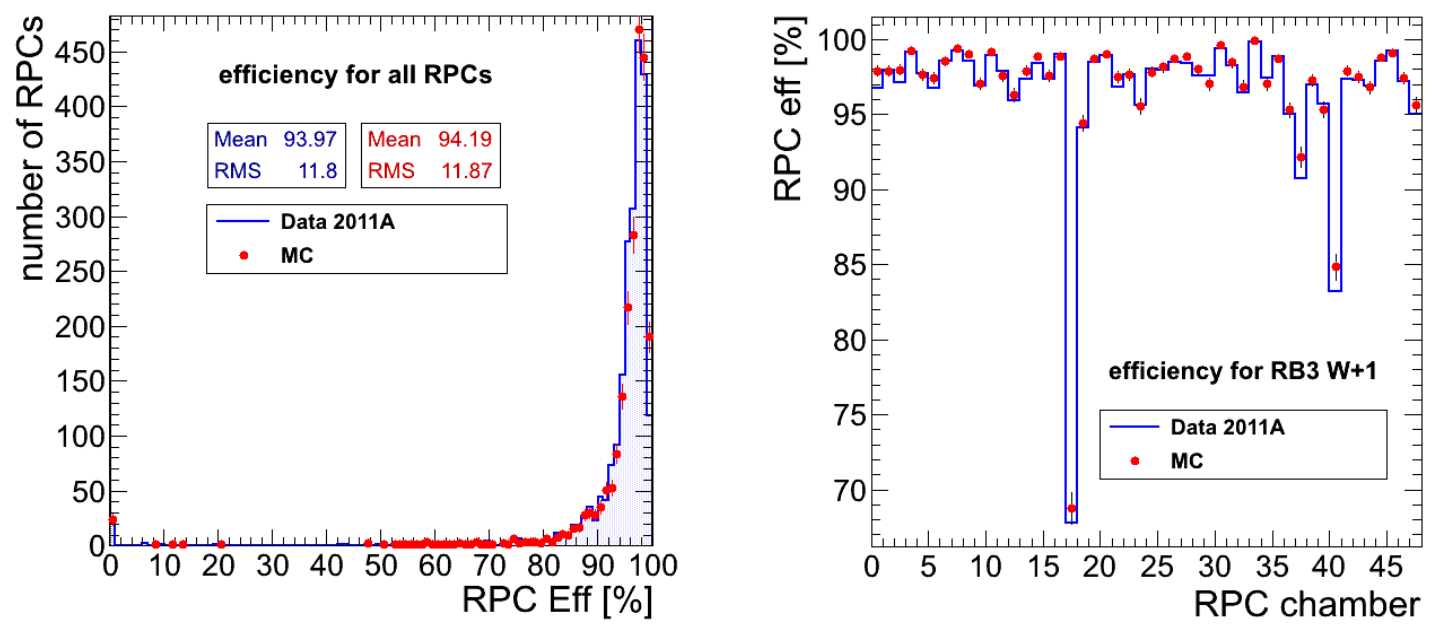

Figure 1: Left plot - efficiency distribution for all the RPCs (the solid line represents the data, the dots the MC results); Right plot - example of the efficiency for all the RB3 chambers of the Barrel Wheel+1 (the solid line represents the data, the dots - the MC results), one of the chamber is working in a single gap mode, which is well reproduced by the MC 
The segment extrapolation method[4] is used to estimate the RPC efficiency from the experimental data. DT/CSC reconstructed segments are extrapolated to RPC strip planes and used to find an RPC hit in an acceptance region around the extrapolated point. Efficiency is computed as the ratio between the observed RPC hits and the expected number of hits from segment extrapolation. The average efficiency computed with the previews method is assigned to all the strips of the same chamber for the simulation purpose. These values are used for the RPC efficiency parametrization. The decision of the algorithm whether the strip will be fired is based on the comparison between the efficiency parameter assigned to the strip and the value taken from the flat distribution.

For the validation purpose the RPC efficiency is estimated as the ratio between the number of events with at least one fired strip corresponding to a simulated muon hit and the total number of simulated muons crossing RPC.

\subsection{Cluster size}

The number of consecutive fired strips (cluster size) is calculated using an empirical cluster size distribution for each chamber. The parametrization was obtained by using data collected from cosmic rays. Moreover, from experimental data resulted that the cluster size depends on the muon impact point relative to the edge of the strip. Therefore the strip is formally subdivided in five slices along its width, and the impact point of the muon is used to find the fired slice coordinates. The number and the position of the adjacent simulated fired strips depend on the slice crossed by the particle. The comparison between the cluster size distributions obtained with experimental and MC data (figure 2) shows a good agreement between the MC parameterization and the experimental data.

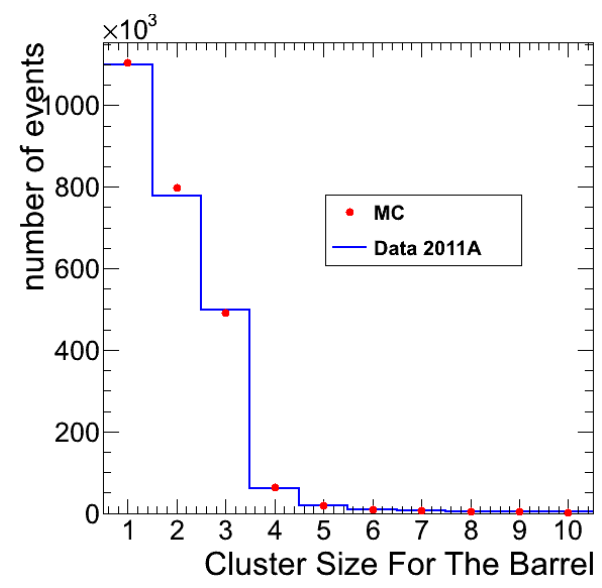

Figure 2: Barrel cluster size for muons crossing RPCs (the solid line represents the data, the dots - the MC results); All stations are merged together

\subsection{Timing}

For proper bunch crossing assignment the time of flight of the particle, the signal propagation speed on the strip and the delay of the signal along the cables for each chamber are taken into account. The time of flight depends on the momentum of the muon and is calculated for each 
simulated particle. The signal propagation time along the strip depends on the hit position on the strip. It is simulated assuming the signal propagation speed is equal to $66 \%$ of the speed of light. The time resolution of the detector is supposed to be Gaussian and simulation is based on the standard normal distribution with sigma of $2.5 \mathrm{~ns}$. To take into account the time response of the detector and relative electronics a constant offset of $50 \mathrm{~ns}$ is added.

\subsection{Noise}

There are two possible types of parametrization used for intrinsic noise simulation. An ideal parametrization where all the strips have the default value of $0.05 \mathrm{~Hz} / \mathrm{cm}^{2}$ was used in the first half of the 2011.

A realistic noise parametrization has been used afterwards. Each strip has its own noise parameter. The intrinsic RPC noise is measured during cosmic runs and used to model the MC response. The measured strip noise $N_{j}$ is used to calculate the average noise rate $v$ per strip:

$$
v=N_{j} S_{s t r} t
$$

where $N_{j}$ is the measured strip noise in $\mathrm{Hz} / \mathrm{cm}^{2} ; S_{s t r}$ is the strip area in $\mathrm{cm}^{2} ; t=n_{b x} 25 n s$, where $n_{b x}$ is the number of simulated bunch crossings.

The estimation of the simulated noise rate for a given strip in a certain event is based on the Poisson distribution with mean $v$. The noise rate is uniformly distributed in time windows around the central bunch crossing including the central one.

The comparison between the noise estimated from experimental data and the simulated noise is shown on the figure 3. For the validation purpose the average simulated noise for the chamber $\bar{N}_{c h}$ is calculated as:

$$
\bar{N}_{c h}=\frac{\sum_{n_{e v}} d_{n o i s e}}{S_{s h} T}
$$
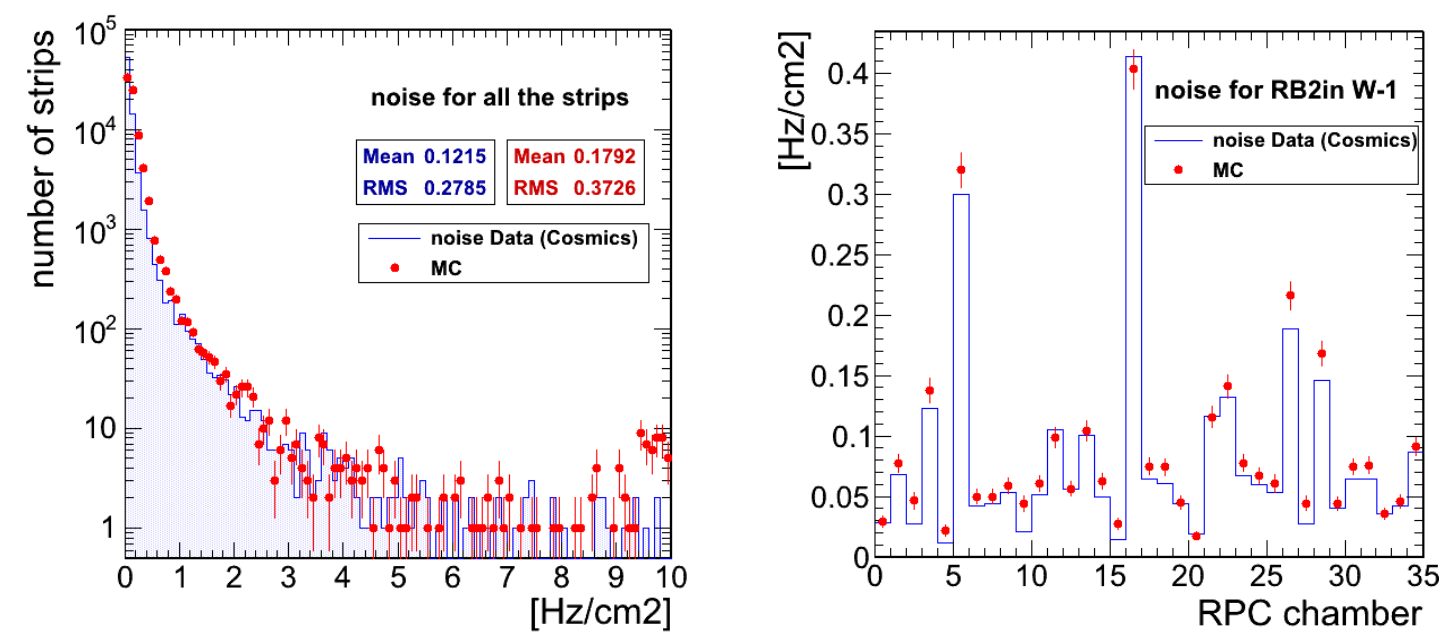

Figure 3: Left plot - Intrinsic noise distribution for all the RPCs strips (the solid line represents the data, the dots - the MC results); Right plot - example of correlation between simulated and measured noise for all the RB2in chambers of the Barrel Wheel-1 (the solid line represents the data, the dots - the MC results) 
where $d_{\text {noise }}$ is the number of all noisy strips for the chamber in the event; $S_{c h}$ - the chamber area; $n_{e v}-$ number of simulated events; $T$ - total time.

\section{Reconstruction validation step}

RPC hits are mainly used as dedicated trigger detector, however they give a contribution to the muon reconstruction as well[5]. The number of RPC hits used in the muon reconstruction is related to the number of RPC stations crossed by the muon (that is a function of $\eta$ ) and of the intrinsic RPC efficiency. The comparison between the average number of reconstructed RPC hits along the muon track for data and MC is shown in figure 4 as a function of $\eta$ and $\varphi$. The shapes of the plots reflect the geometrical details of the RPC muon system and the presence of dead chambers. The simulation model well reproduces both features.
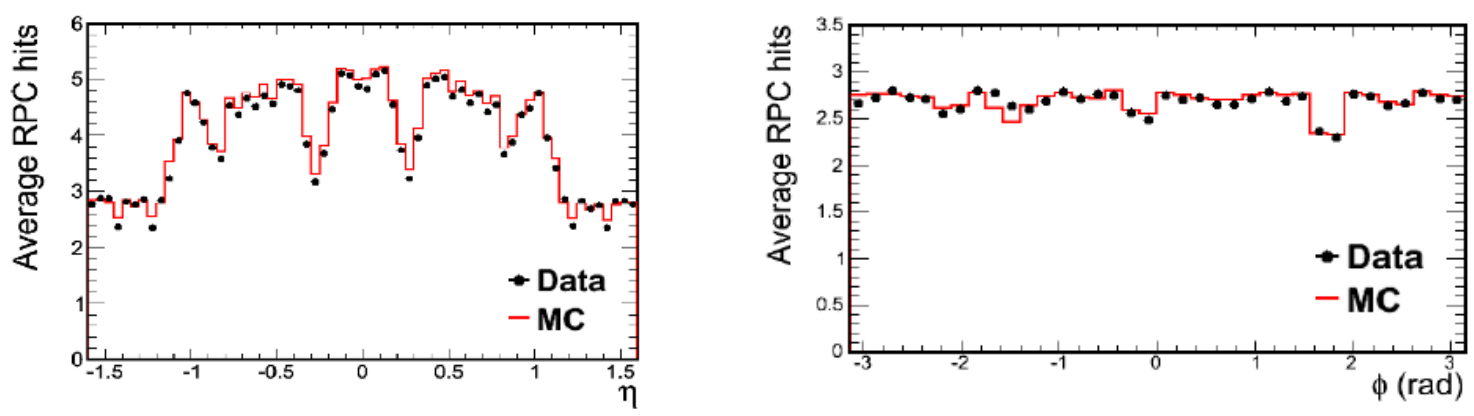

Figure 4: Left plot - The average number of RPC hits associated to the muon as function of $\eta$ for Global Muons with $P_{T}>20 \mathrm{GeV} / \mathrm{c}$ coming from $Z$ decay (the dots represents the data, the solid line - the MC results); Right plot- The average number of RPC hits associated to the muon as function of $\varphi$ for Global Muons with $P_{T}>20 \mathrm{GeV} / \mathrm{c}$ coming from $Z$ decay (the dots represents the data, the solid line - the MC results).

\section{Conclusion}

An algorithm for simulation of the RPC response is implemented in the CMSSW (CMS software framework). The algorithm uses a set of parameters, estimated from the real data. The set of used parameters is regularly updated according to the experimental data. A dedicated validation procedure is developed. The simulation is in good agreement with the experimental data. The reliability of the RPC response simulation with realistic parameters is shown.

\section{Acknowledgments}

The speaker would like to thank to Davide Piccolo, Camilo Carrilo, Marcelo Abbrescia, Borislav Pavlov and Leandar Litov for the useful discussions and to the whole RPC group at CMS experiment for having provided important experimental information. This work is supported by Ministry of Education, Youth and Science, Bulgaria under contract D01-757/20.12.2008. 


\section{References}

[1] CMS Collaboration, The Muon Project Technical Design Report, CERN/LHCC 97-32 (1997);

[2] M. Abbrescia et al., Cosmic ray tests of double-gap resistive plate chambers for the CMS experiment, Nucl. Instrum. Meth. A 550 (2005) 116;

[3] S. Agostinelli and et al. (Geant4 Collaboration), Geant4 a simulation toolkit, Nucl. Instr. and Meth. A 506 (2003) 250;

[4] Camilo Carrillo, The CMS RPC project, results from 2009 cosmic-ray data, Nucl. Instrum. Meth. A 661 (2012) S19-S22;

[5] M. Kim, The RPC hits in the CMS muon reconstruction, these proceedings PoS (RPC2012) 045 ; 